\title{
Prioritized Energy-Efficient MAC Protocol in Wireless Sensor Networks
}

\author{
Hyun Joo Park ${ }^{\mathrm{a}, *}$ \\ ${ }^{a}$ Department of Smart Information and Telecommunication Engineering, Sangmyung University, 31066, Cheonan, Korea \\ Corresponding author: * cathy2369@smu.ac.kr
}

\begin{abstract}
In this paper, we propose a MAC protocol that provides energy efficiency and QoS for wireless sensor networks. Because it does not classify the data according to the characteristics, traditional MAC protocols for Wireless Sensor Networks cannot provide service differentiations for different traffic classes. For the purpose of QoS support, the input data to the node is divided into priority classes according to the transmission emergency. Depending on the urgency of the data, the protocol can provide time slots for nodes and take different contention window sizes for different traffic classes. Nodes use the reinforcement learning-based control mechanism to actively infer the status of other nodes to classify effectively under various kinds of traffic conditions. The proposed protocol can meet the QoS requirements of delay-sensitive applications and can effectively deal with the problem of a multi-channel hidden terminal with the use of multi-queue. For the next purpose, the proposed protocol is energy efficient because it is based on sleep mode. To improve the energy efficiency, each node has a short non-overlapping listening interval, saving energy in idle listening intervals. This is one of the ways to efficiently use the energy of a node to extend the life of the entire network. This achieves low power consumption by increasing the throughput of data processed across the network and enabling efficient transmission. The classified data can be further improved by rearranging the data according to the nature of the transmitted data in the queue.
\end{abstract}

Keywords - QoS; energy-efficient MAC protocol; delay; network lifetime; large amount of data; wireless sensor networks.

\section{INTRODUCTION}

Wireless Sensor Networks are widely applied in various fields such as environmental monitoring, natural disaster management, management in logistics, home network management, animal management in the farm, object tracking, telematics in the transportation, and hospital patient management in the medical field. Since the data collected during environmental monitoring is collected for various purposes, quality assurance of emergency is an important consideration [1]-[3]. Also, the energy efficiency of a batteryoperated sensor affects the life of the entire network, so it must be considered [4] [3] [5]. Figure 1. shows the application fields of the wireless sensor network. Recently, wireless networks are increasingly used in logistics and telematics.

An important role of Wireless Sensor Networks in these applications is to monitor the target area [6] and deliver data collected from each sensor node [5]. Typically, Wireless Sensor Networks consist of many sensor nodes that make up their multi-hop wireless network. Each sensor node communicates with the other using a low power signal. Unlike other wireless networks, however, efficient energy use of each sensor node is required to extend the overall network life of a battery-powered wireless sensor network [7], [8].

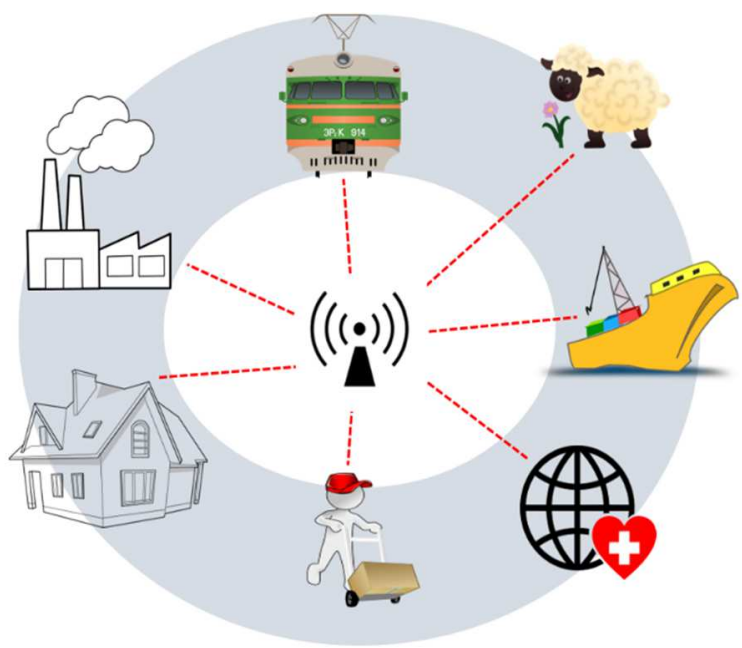

Fig. 1 Various fields of Wireless Sensor Networks 
Idle listening of sensor nodes is a waste of energy. Therefore, several studies have been proposed to prevent energy consumption by reducing idle listening. Some studies have been conducted on how to save energy by allowing sensor nodes to turn off their radio periodically and stay in sleep whenever possible [8], [9]. Most of these findings have the effect of saving energy by increasing the listening interval and the sleep interval as much as possible but increasing the delay of the transmitted data [1], [9]. Because the existing MAC protocol was interested in only saving transmission energy, the algorithm considering the redundancy packet and priority of data took over the role by adjusting the contention window size, which has a small processing level or limits the transmission time [4], [6]. The proposed Mac protocol classifies the transmitted data through reinforcement learning at the node and loads multiple queues [6], [10], [11]. If nodes have the same data contents or destination, the transmission priority is made very low to increase transmission efficiency [12]-[14]. In this way, the total traffic can be efficiently operated to extend network life [4]. It is expected that the operation of the entire network will run smoothly by reducing the load on the sink node for energy efficiency and data processing only by efficient operation of transmitted data [11], [15].

An important issue to be addressed in Wireless Sensor Networks is the support of Quality of Service (QoS). The data response priority of the wireless sensor network can be classified into high priority data and non-high priority data, and normal data that is not a high priority is a large amount of data [14], [16], [17]. Bring for this reason. We propose protocols to meet the requirements of both aspects simultaneously. Priority transmission of data generated in real-time and smooth transmission of large-capacity data without priorities can be performed simultaneously to improve the quality of Wireless Sensor Networks to improve the QoS of wireless networks [12], [18], [19].

The proposed MAC is energy efficient and QoS supported multi-layer QAML-MAC (QoS Aware Multi-layer MAC) protocol. The proposed MAC protocol has the advantage of reducing the delay, which is a problem of the previously proposed protocol, and transmitting the high-priority data quickly, thereby transmitting the emergency data first. In addition, if priority data to be transmitted from each node does not exist, multiple layers of idle listen are used to reduce collisions in data transmission, thereby reducing energy consumption in each node and further reducing overall network life.

To this end, data input to the node is divided into priority classes and stored according to transmission emergency. In addition, the concept of cross-layer was introduced to rearrange data destined for the same destination. The proposed MAC protocol has the advantage of reducing the delay, which is a problem of the previously proposed protocol, and transmitting the high-priority data quickly, thereby transmitting the emergency data first. Besides, if priority data to be transmitted from each node does not exist, multiple layers of idle listen are used to reduce collisions in data transmission, thereby reducing energy consumption in each node and further reducing overall network life.

Energy Consumption is the main research field for Wireless Sensor Networks, so MAC protocols take up the requirements of related applications and consider the network's energy consumption. SMAC [20] is the first MAC protocol for energy-saving requirements of Wireless Sensor Networks. By using the duty cycle mechanism, SMAC [20] reduces idle listening, drops overhearing. Using control packets data exchange mechanism guarantees data integrity and reduces the latency of multi-hop transmission by using adaptive listening.

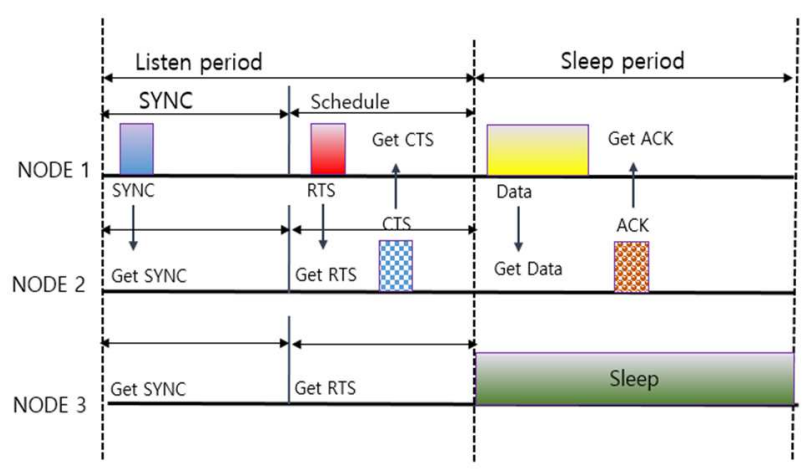

Fig. 2 Data transmission process in SMAC

Fig. 2 shows data transmission process in SMAC. When node A has data to send, it sends a SYNC packet to the neighboring node $\mathrm{B}$ and node $\mathrm{C}$ and sends a request-to-send (RTS) packet to the recipient node B. Node B, which has received the RTS packet, sends CTS in response, and then receives data in the sleep section. However, node $C$ continues to sleep during the sleep period. Since nodes that do not participate in data transmission stay in the sleep mode, energy consumption caused by idle listening can be prevented. However, the SMAC [20] protocol uses the above mechanism to save energy, but it consumes more energy because it has a fixed listen to section even when there is no data due to the fixed listen and sleep intervals.

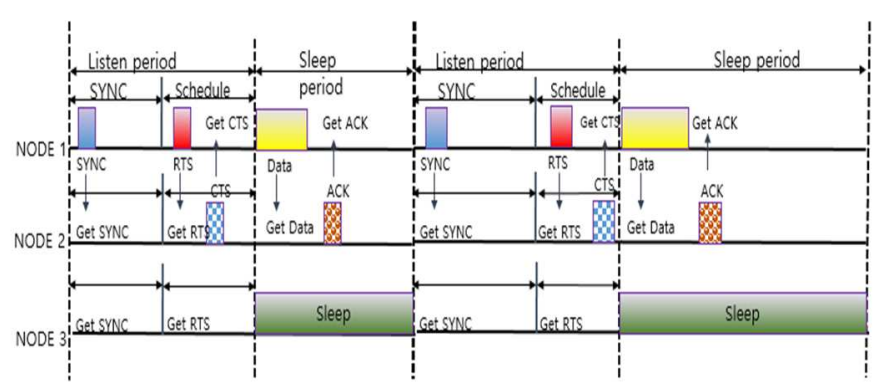

Fig. 3 Data transmission process in TMAC

In this way, the TMAC [8] protocol was proposed to save energy by going to sleep mode when there is no data transmission in the listen to section. However, both SMAC and TMAC protocols have the advantage of efficiently using energy but have a problem in that the delay in packet transmission is increased. Besides, since the sender and receiver can have different schedules and follow FIFO scheduling, additional delays may occur [9].

The EQ-MAC proposed [10] supports QoS through classifier MAC and channel access MAC that classify traffic according to priority. The proposed EQ-MAC is a clusterbased MAC protocol, and the decision of nodes to transmit is contention-based. However, in the data transmission, the 
cluster head uses a TDMA scheme in which the cluster head allocates slots. However, since the EQ-MAC is basically applicable only to the one-hop structure between the sensor nodes and the head node, it is difficult to accurately apply to actual Wireless Sensor Networks composed of multiple hops.

A protocol that integrates energy-efficient use and QoS support, a problem in Wireless Sensor Networks, has been proposed [12], [18], [19]. The system proposed [18] is an integrated wireless transmission system that combines QoS requirements from the application layer with modulation and transmission schemes in the data link and physical layers. In addition, the protocol proposed here is divided into intracluster and inter-cluster on a cluster basis. The cluster head thus forms a multi-hop backbone. However, there is a problem that the performance affects a lot depending on the values of the parameters used.

In addition, the Q-MAC structure proposed [12], [19] supports QoS by differentiating network services based on priorities. The difference in priority level here is determined by reflecting the emergency of the data measured from different sensor nodes. To this end, intra-node scheduling and external inter-node scheduling are used. The use of the priority buffer in this paper follows the method of intra node scheduling of Q-MAC. The QAML-MAC proposed in this paper is the same as the Q-MAC in that it transmits priority according to the packet emergency. However, there is a difference in that the transmission order is changed according to the packet's destination. Different transmission schedules have the advantage of reducing energy efficiency and delay.

\section{MATERIALS AND MeTHOD}

The proposed protocol can make differentiated transmission by defining QoS priority according to the transmission emergency of each data while extending the life of the whole network with efficient use of energy. In addition, multi-scheduling according to the distance between the Sink node and the cross-layer method of separately storing and transmitting the transmitted data packet to speed up the burst traffic of data requires real-time transmission such as multimedia traffic. Based on the requirements of network applications, QAML-MAC determines the time slot's proportions about different traffic classes.

QAML-MAC is a kind of QoS-Aware MAC protocol for network applications with multi-traffic classes, using a periodic wake-up mechanism. As shown in Fig. 4, MAC is composed of SYNC phase, scheduling phase, and data phase. In SYNC phase, by the exchange of the sync data packets, the virtual cluster is formed. To avoid collisions in data transmission, all nodes in the same virtual cluster need to broadcast their own scheduling information regularly. In the scheduling phase, the sender node and the receiver node are required to complete the time slot scheduling by exchanging the RTS/CTS control frame. At this phase, the number of timeslots is set.

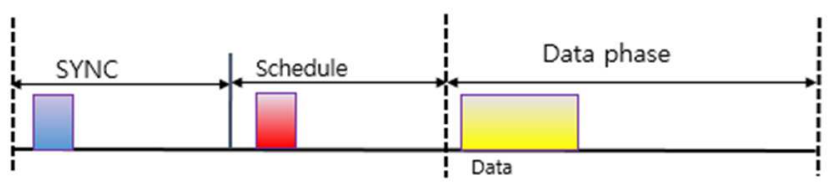

Fig. 4 Periodic wake-up mechanism of QAML -MAC
We propose an integrated MAC protocol combining the methods. In the proposed QAML-MAC protocol, nodes have a schedule for transmitting priority data and two transmission schedules. Therefore, in QAML-MAC protocol, it is stored in an independent queue according to the priority determined according to the transmission emergency and has the advantage of reducing the transmission delay by transmitting data to the same destination at the time of storage. Besides, the newly created data is first transmitted by occupying the channel with CSMA method by increasing the priority, and the general data near the Sink node is transmitted by the TDMA method to increase the transmission efficiency.

\section{A. QAML-MAC Protocol Transmission Mechanism}

The QAML-MAC protocol proposed in this section is competition-based and consists of the following two elements. The first is to classify the priority classes of the data and store them in the buffer of each class. In this case, the data destined for the same destination is arranged adjacently using the cross-layer concept. The second method is to allocate the sensor nodes layered to not overlap by dividing the listen to interval to reduce energy consumption in the listen to the interval.

First, each sensor node classifies the received data and stores them in each buffer. Figure 5. below shows the mechanism for storing the data received from neighbor nodes in separate buffers for each priority [6]. As shown in the figure, packets received from neighboring nodes are stored in the priority classifier, and the lower priority buffer in the packet classifier according to the emergency of the packet and are delivered to the next node in order.

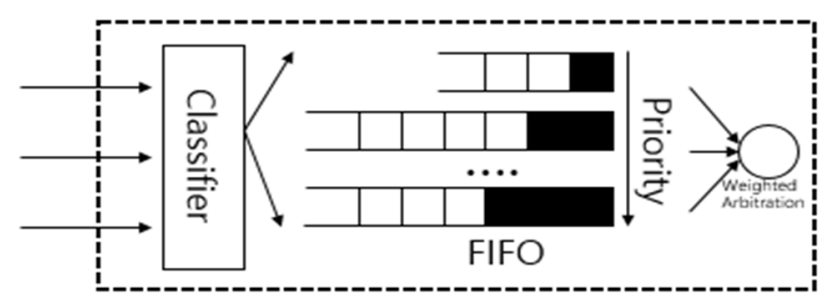

Fig. 5 Node separate buffers for each priority

To distinguish the data class, each sensor node prioritizes its measured value or transmission urgency [6] of data received from neighboring nodes. Transmission urgency $\mu$ is the packet urgency $(\mathrm{Cc})$ based on the importance of the application layer, transmission hops $(\mathrm{Hc})$ indicating the retransmission cost, residual energy (Ec), and queue's proportional load. The relationship is as follows.

$$
\begin{aligned}
& \mu=\frac{1}{4} \times\left(\frac{E_{c}}{E_{\ldots \ldots} \ldots}+\lambda+\frac{c_{c}}{c_{\ldots} \ldots}+\frac{H_{c}}{H_{\ldots} \ldots}\right) \\
& \lambda=\frac{1}{2} \times\left(\frac{\sum_{i=1}^{n} \omega_{i} Q_{c}(i)}{\sum_{i=1}^{n} \omega_{i} Q_{(i)}}+\max _{k}=1 \ldots n\right)
\end{aligned}
$$

\section{B. QAML-MAC Protocol Slot Scheduling Mechanism}

The node can schedule by exchanging RTS/CTS control frame in the scheduling phase. QAML MAC divides the alltime slot phase into two parts. High priority data part and low priority data part. High priority data part can use more priority 
data part slots. Data slot's length is different, it depends on the priority queue [2], [11], [19].

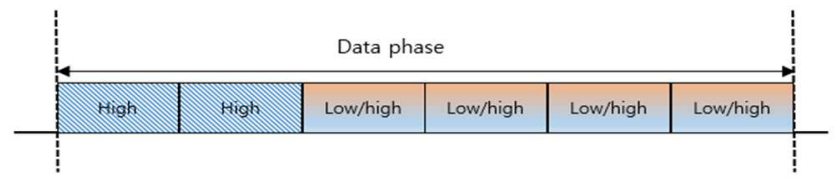

Fig. 6 Case with more low priority Data

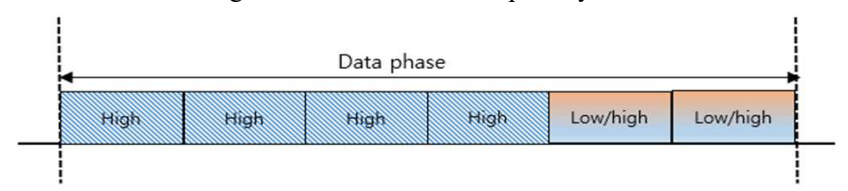

Fig. 7 Case with more high priority Data

When duplicate data is detected, data is transmitted by increasing the transmission cycle. By increasing the transmission period of the data accumulated in the buffer, less data is transmitted during the same time. In this way, the network increases efficiency. Reinforcement learning operations queue data collected from nodes. We use RL-MAC operation that adjusts the length of the appropriate queue. The packet has fields that provide information on the success or failure of node node transmission to the header and uses them to adjust the queue's length. In the RL-MAC, the length of the queue is adjusted by referring to the successfully transmitted packet and the packet number of failed transmission attempts by the sender.

The priority of incoming Packets determined by the content of the packet and traversed hop count. In this way, packets are mapped into predefined different priority queues. Using the MAX-MIN fairness algorithm [19], control the rate and packetized for Intra node scheduling. To select the next transmitted packet, Generalized Processor Sharing [11] algorithm is used. For inter-node scheduling, a new method called Loosely Prioritized Random Access (LPRA) is proposed to adjust the media access order of nodes with packets transmitted based on transmission urgency.

\section{QoS Support in QAML-MAC Protocol}

Depending on the destination of the node, the transmission data order is changed to reduce the transmission energy of the entire network. That is, the delay problem caused by FIFO scheduling is solved by changing the transmission order according to the destination of data transmitted from one node. Each node creates a temporary buffer to reverse the order of the data according to the destination and to send packets destined for the same destination first [6], [19].

When data is collected from a node, data is classified using the information in the header [12]. The information included in the header is the location of data generation and a time destination address. Using this information, the information generated simultaneously at the same location is regarded as duplicate data. Avoiding redundant data transmission can be a good way to reduce data traffic. Besides, if data originating from the same location has different destinations, the transmission priority is high data. Among them, data is allocated to the queue with the highest priority.

When classifying the node data in the RL-Classifier, the priority is divided through reinforcement learning and stored in the queue [1]. Priority is given differently depending on the urgency and traffic characteristics of the node [11], [16].

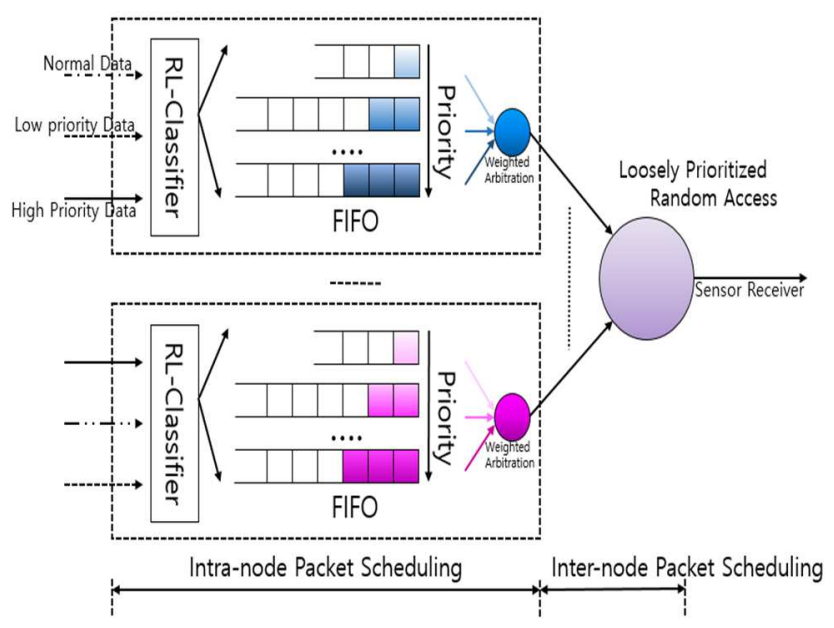

Fig. 8 QAML-MAC Scheduling Inter node \& Intra node

\section{Energy Saving in QAML-MAC Protocol}

The method of reducing the listen section to save energy of the sensor node can extend the life of the entire network, but it is not good method because it reduces the reliability of the network due to data transmission failure. This is a good way to increase network reliability and overall lifetime. It belongs to multiple sections that do not overlap the listen section for energy-efficient use [9], [18]. As shown in Fig.5, each node has a short, non-overlapping listen interval, saving energy in the idle listening interval. Therefore, as shown in Fig.3, each node has a non-overlapping listen interval, thus saving energy that can occur in the listen interval.

When there is no priority data to be transmitted in each node, multiple layers are used for idle listen, thereby reducing collisions in data transmission, and reducing energy consumption at each node. The transmission of no priority data is operated by applying a layered method to save energy. By operating the layered active section instead of turning off the radio of the sensor node, energy is saved and data to be transmitted is not missed at the same time.

One fixed frame is divided into listen section and sleep section. Listen section is to save energy, it operates in a multilayer so that the active state of the listen section does not overlap, which has more energy-saving effect than having the active state in the entire listen section. Since the proposed protocol is designed to operate in muilti-hop, it is possible to operate a layered method between listen sections with other nodes. Fig. 9 shows how to save energy with operating multilayer listen sections.

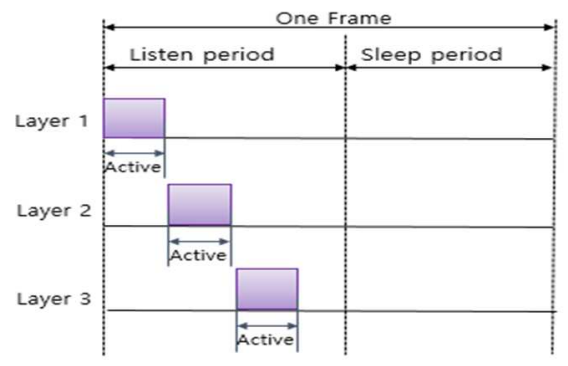

Fig. 9 Save energy by layering listen to sections 


\section{E. Algorithm}

QoS support of the QAML-MAC protocol is performed in the following order.

- Each sensor node has a schedule with non-overlapping listen to intervals and a schedule that all nodes have in common, such as SMAC or TMAC protocols.

- When each node transmits data of a high priority buffer, In the sync interval, all nodes have a common schedule. All nodes with this schedule will synchronize to this schedule. Thus, all nodes operate with the TMAC protocol. In this case, however, each buffer is rearranged, stored, and transmitted to the same destination.

- When transmitting low-priority data from each node, follow the schedule of multiple listen layers.

- Operates with CSMA or TDMA depending on the value of the packet priority variable.

\section{RESULT AND DISCUSSION}

The performance of the proposed QAML-MAC protocol is compared with that of the existing MAC. Since it has the same listen-sleep structure as the existing MAC, it is mainly a scheduling part of packet transmission in comparison to delay. However, the QAML-MAC protocol extends the entire network's lifetime by reducing energy consumption at each node by supporting QoS in response to data transmission urgency, using fewer listen to intervals, and reducing the probability of collision. The parameters used in the networks are shown in Table I. For the following performance comparison, it is assumed that $30 \%$ of the total data transmitted by each node has priority traffic, that is, real-time traffic such as multimedia or abnormal data exceeding an initially set threshold.

TABLE I

NETWORKING PARAMETERS FOR SIMULATION

\begin{tabular}{lll}
\hline \multicolumn{1}{c}{ PARAMETER } & \multicolumn{1}{c}{ DESCRIPTION } & \multicolumn{1}{c}{ VALUE } \\
\hline Duty Cycle & & 10 \\
Data_CW & Maximum Window Size & 63 \\
Short_CW & Minimum Window Size & 31 \\
Pt & Transmit Power Consumption & 0.2818 \\
Pr & Receive Power Consumption & 0.0 .3682 \\
Pidle & Idle Power Consumption & 0.3442 \\
Psleep & Sleep Power Consumption & 0.00005 \\
Pinit & Initial Energy & 1000 \\
\hline
\end{tabular}

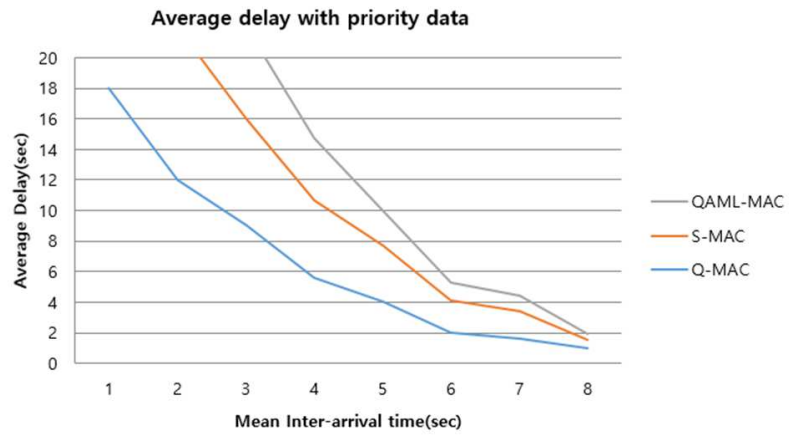

Fig. 10 Average delay with priority Data

As shown in Fig. 10, it can be seen that the QAML-MAC protocol proposed in this paper has a low average delay for data with priority. Since the SMAC protocol basically does not support priority, it represents the average delay of all transmitted data.

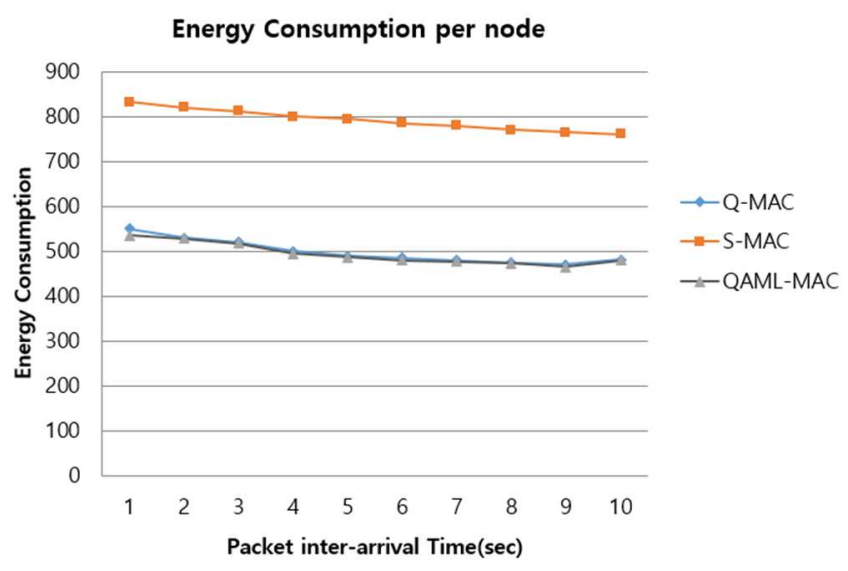

Fig. 11 Energy Consumption per Node

Fig.11 shows a comparison of the average energy consumed by one node. The QAML-MAC protocol using multiple layers shows that the amount of energy consumed per node is significantly lower than that of the SMAC protocol. This is because energy consumption in the listen section is low, and energy is saved by reducing collisions of transmitted packets.

\section{CONCLUSION}

The proposed MAC protocol reduces delay, which is a problem of the proposed protocol and provides high priority data such as data exceeding a threshold in applications such as real-time multimedia traffic or predetermined field monitoring. The fast transmission has the advantage of transmitting emergency data first. In addition, by using the idle listening section divided into several layers, the collision of transmitted packets can be reduced, thereby reducing the energy consumption at the node and increasing the overall network life. The QAML-MAC protocol extends the life of the entire network by reducing energy consumption at each node by supporting QoS according to data transmission emergency and reducing the use of fewer listen sections and the probability of collision. In order to improve the accuracy of QAML-MAC proposed in this paper, the study on the setting of each parameter will be done later.

\section{ACKNOWLEDGMENT}

This research is supported by a 2019 Research Grant from Sangmyung University.

\section{REFERENCES}

[1] I. Villordo-Jimenez, N. Torres-Cruz, M. M. Carvalho, R. MenchacaMendez, M. E. Rivero-Angeles, and R. Menchaca-Mendez, "A Selective-Awakening MAC Protocol for Energy-Efficient Data Forwarding in Linear Sensor Networks," Wirel. Commun. Mob. Comput., vol. 2018, pp. 1-18, 2018, doi: 10.1155/2018/6351623.

[2] A. K. Subramanian and I. Paramasivam, "PRIN: A Priority-Based Energy Efficient MAC Protocol for Wireless Sensor Networks Varying the Sample Inter-Arrival Time," Wirel. Pers. Commun., vol. 92, no. 3, pp. 863-881, Feb. 2017, doi: 10.1007/s11277-016-3581-5.

[3] A. More and V. Raisinghani, "A survey on energy efficient coverage protocols in wireless sensor networks,"J. King Saud Univ. - Comput. 
Inf. Sci., vol. 29, no. 4, pp. 428-448, Oct. 2017, doi: 10.1016/j.jksuci.2016.08.001.

[4] J. Lee and S. Kim, "EnRI-MAC: an enhanced receiver-initiated MAC protocol for various traffic types in wireless sensor networks," Wirel. Networks, vol. 26, no. 2, pp. 1193-1202, Feb. 2020, doi: 10.1007/s1 1276-018-1854-5.

[5] A. Kochhar, P. Kaur, P. Singh, and S. Sharma, "Protocols for Wireless Sensor Networks: A Survey,” J. Telecommun. Inf. Technol., vol. 1, pp. 77-87, Apr. 2018, doi: 10.26636/jtit.2018.117417.

[6] H. J. Park, S. C. Kim, and H. Y. Kim, "Priority Aware Data Collection Mac Protocol in Wireless Sensor Networks," Int. J. Adv. Sci. Technol., vol. 124, pp. 69-78, Mar. 2019, doi: 10.33832/ijast.2019.124.06

[7] Z. Li, Y. Liu, M. Ma, A. Liu, X. Zhang, and G. Luo, "MSDG: A novel green data gathering scheme for wireless sensor networks," Comput. Networks, vol. 142, pp. 223-239, Sep. 2018, doi: 10.1016/j.comnet.2018.06.012.

[8] T. van Dam and K. Langendoen, "An adaptive energy-efficient MAC protocol for wireless sensor networks," in Proceedings of the first international conference on Embedded networked sensor systems SenSys '03, 2003, p. 171, doi: 10.1145/958491.958512.

[9] Z. Tang and Q. Hu, "An Adaptive Low Latency Cross-Layer MAC Protocol for Wireless Sensor Networks," in 2009 Eighth IEEE International Conference on Dependable, Autonomic and Secure Computing, Dec. 2009, pp. 389-393, doi: 10.1109/DASC.2009.53.

[10] K.-J. PAEK, J. KIM, U.-S. SONG, and C.-S. HWANG, "PriorityBased Medium Access Control Protocol for Providing QoS in Wireless Sensor Networks," IEICE Trans. Inf. Syst., vol. E90-D, no. 9, pp. 1448-1451, Sep. 2007, doi: 10.1093/ietisy/e90-d.9.1448.

[11] K. Shim and H.-K. Park, "Priority-Based Pipelined-Forwarding MAC Protocol for EH-WSNs," Wirel. Commun. Mob. Comput., vol. 2019 pp. 1-7, May 2019, doi: 10.1155/2019/5418516.

[12] S. Sarang, M. Drieberg, A. Awang, and R. Ahmad, “A QoS MAC protocol for prioritized data in energy harvesting wireless sensor networks," Comput. Networks, vol. 144, pp. 141-153, Oct. 2018, doi: 10.1016/j.comnet.2018.07.022.

[13] S. C. Kim, H. J. Park, and H. Y. Kim, "Improving the Network Lifetime of WSN Using An Intelligent Data Collection MAC Protocol," JP J. Heat Mass Transf., no. Special, pp. 99-106, Nov. 2019, doi: 10.17654/HMSI1 19099.

[14] F. Alfouzan, A. Shahrabi, S. Ghoreyshi, and T. Boutaleb, "An Efficient Scalable Scheduling MAC Protocol for Underwater Sensor Networks," Sensors, vol. 18, no. 9, p. 2806, Aug. 2018, doi: 10.3390/s18092806

[15] X. Yang, L. Wang, J. Su, and Y. Gong, "Hybrid MAC Protocol Design for Mobile Wireless Sensors Networks," IEEE Sensors Lett., vol. 2 , no. 2, pp. 1-4, Jun. 2018, doi: 10.1109/LSENS.2018.2828339.

[16] F. Rismanian Yazdi, M. Hosseinzadeh, and S. Jabbehdari, "A PriorityBased MAC Protocol for Energy Consumption and Delay Guaranteed in Wireless Body Area Networks," Wirel. Pers. Commun., vol. 108, no. 3, pp. 1677-1696, Oct. 2019, doi: 10.1007/s11277-019-06490-z.

[17] K. Oh, G. Gim, W. Lim, and B. M. Kim, "An Energy-Efficient and Collision-Avoidance MAC Protocol with Pre-scheduling for Wireless Sensor Networks," Wirel. Pers. Commun., vol. 102, no. 1, pp. 61-78, Sep. 2018, doi: 10.1007/s11277-018-5825-z.

[18] G. Zheng, Y. Sun, B. Kang, H. Ma, J. Li, and Y. Wang, “A QoS-aware MAC protocol for Wireless Sensor Networks," Int. J. Comput. Sci. Issues, vol. 14, no. 1, pp. 1-8, Jan. 2017, doi: 10.20943/01201701.18.

[19] H. Y. Kim, S. C. Kim, and H. J. Park, "Priority Aware MAC Protocol for Delay-Bounded Applications in Wireless Sensor Networks," Int. J. Grid Distrib. Comput., vol. 11, no. 10, pp. 41-50, Oct. 2018, doi: 10.14257/ijgdc.2018.11.10.04.

[20] W. Ye, J. Heidemann, and D. Estrin, "Medium Access Control With Coordinated Adaptive Sleeping for Wireless Sensor Networks," IEEE/ACM Trans. Netw., vol. 12, no. 3, pp. 493-506, Jun. 2004, doi: 10.1109/TNET.2004.828953. 